\title{
Diets of obese and non-obese children
}

\author{
Atsuko Satoh $^{1^{*}}$, Seiko Fujita ${ }^{2}$, Kazuko Menzawa $^{3}$, Sangun Lee $^{4}$, Masao Miyamoto $^{1}$, Hidatada Sasaki $^{5}$ \\ ${ }^{1}$ Akita University of Nursing and Welfare, Odate, Japan; *Corresponding Author: a-satoh@well.ac.jp \\ ${ }^{2}$ Hiroaki Kensei Hospital, Hirosaki, Japan; \\ ${ }^{3}$ Faculty of Education, Hirosaki University, Hirosaki, Japan; \\ ${ }^{4}$ Department of Physical Therapy, Aomori University of Health and Welfare, Aomori, Japan; \\ ${ }^{5}$ Sendai Tomisawa Hospital, Sendai, Japan.
}

Received 23 March 2011; revised 8 May 2011; accepted 20 July 2011.

\begin{abstract}
Aim: To compare diets between obese and nonobese in children. Methods: Thirty-four obese and ten non-obese school children were recruited and their habitual factors of obesity were asked. Intakes of food in the obesity and non-obesity groups were checked using a model nutritional balance chart (MNBC). Results: Average intake ratio of food relative to ideal food intake was significantly higher in the non-obesity group than the obesity group. The relationship between obesity and exercise was significant but not significant for intake ratio of food, times watching TV and playing games. Conclusion: Food intake is not a primary factor of obesity but exercise is a key factor for obesity in school children. Since the effect of diet intervention in obese children was slight, exercise habit would be a more important strategy to reduce obesity than diet in school children.
\end{abstract}

Keywords: Food Intake; Exercise; Sedentary Times; Children; Obesity

\section{INTRODUCTION}

In the last 20 years, the prevalence of obesity increased two-fold (from 5\% to 10\%) in school children in Japan [1]. Childhood obesity is an urgent issue to subside increasing metabolic syndrome in adulthood because obesity in children tends to persist into adulthood. Interventions to prevent childhood obesity resulted in not consistent or limited effects of dietary habits and physical activity [2]. Physical activity in children above the government-recommended intensity was not associated with a change in obesity [3]. Cross-sectional studies showed that children's weights were associated with food consumption [4] and sedentary activities [5]. However, it has not been reported which is the key factor to contribute obesity. In the present report, we studied diets between obese and non-obese children and compared diets with physical activity, time watching TV and playing games.

\section{METHODS}

The subjects were recruited from primary school children and a junior high school in Odate City, Akita prefecture, Japan, where the authors had school health assignments as nurses. A child was considered to be obese when the body weight exceeded $120 \%$ of the standard body weight, which is defined as the mean body weight corresponding to the height for that age and sex obtained from national statistics for Japanese school children in 2009 [1]. The criteria for inclusion as non-obese children are body weight less than $120 \%$ of the standard body weight. The subjects of the present study had no endocrine, metabolic or kidney disease. Among 43 obese children, 9 children refused to participate in the study, leaving 34 children female/male, 20/14, average age $11.9 \pm 1.5$ years, and ranging from $8-14$ years. Fifteen children were randomly chosen for the non-obese group. Five children in the non-obese group refused to participate in the study leaving 10 children (female/male, 5/5, average $11.7 \pm 2.3$ years, ranging from 7 to 14 years) in the non-obese group. Among the 10 non-obese children, 5 were in primary school and 5 were in junior high school. It took one year of serial participation by the subjects, from August 2003 to July 2004.

All the participants, including their parents, completed a questionnaire about physical characteristics, health assessment, life styles, such as exercise habits and time watching television and playing games, family composition, and eating habits. Exercise habits were defined as participating in sporting clubs such as football, basketball, baseball and so on associated with the school or outside school and practiced exercise at least twice a week. Food intakes were asked using the model nutri- 
tional balance chart (MNBC) as described previously and explained briefly here [6]. The MNBC was designed to outline a $6697 \mathrm{kj}$ intake according to the recommendation by the Japan Obesity Society [7]. The MNBC demonstrates the ideal dietary distribution of 11 categories of food: meat, fish, eggs, milk and dairy products (hereinafter called milk), beans and bean products, such as bean curd and miso soup (hereinafter called beans), green and yellow vegetables, light-colored vegetables, fruit, potatoes and grains (hereinafter called grains), oil, and sugar. The number of times each food category was consumed was marked with black dots; the foods eaten were recorded by category, but not by amount. The child (and/or mother) filled out a meal chart which consisted of columns for breakfast, lunch, dinner, and snacks between meals. The meal chart was to be filled out for 3 days of the last week of one month; Friday, Saturday, and Sunday. We calculated the nutritional balance, as follows: (intake ratio of food $=$ the actual food intake [black dots] $\div$ the ideal food intake following the MNBC [black dots]). Thus the nutritional balance based on the MNBC was ideally “ 1 ”. Ethical approval was obtained from the Ethical Committee of the University. The Mann-Whitney's U test was used for the significance.

Multivariate logistic regression was used to determine the variations. Values were presented as mean \pm SD. Statistical significance was considered for $p<0.05$.

\section{RESULTS}

Percentage overweight values of obese children and non-obese children were $50 \pm 21 \%$ and $-3 \pm 8 \%$, respectively. The numbers of children with exercise habits and without exercise habits were 9 and 25 in the obesity group and 8 and 2 in the non-obesity group, respectively. Intake-ratio of food in the obesity group was significantly lower than that of the non-obesity group $(p<0.01)$ (Table 1). Exercise performance was significantly higher in non-obese children than obese children $(p<0.01)$. Children with exercise showed a significantly lower degree of obesity than children without exercise (11 $\pm 20 \%$ vs. $56 \pm 23 \%)(p<0.01)$. Time watching TV was significantly lower in non-obese children $(2.0 \pm 0.9 \mathrm{~h})$ than obese children $(3.0 \pm 1.5 \mathrm{~h})(p<0.05)$. Time playing games was significantly lower in non-obese children $(0.1$ $\pm 0.3 \mathrm{~h})$ than obese children $(0.4 \pm 0.8 \mathrm{~h})(p<0.05)$. The children with exercise showed significantly shorter times of summation of watching TV and playing games than the children without exercise $(2.7 \pm 1.2 \mathrm{~h}$ vs. $3.2 \pm 2.3 \mathrm{~h})$ $(p<0.01)$. The relative impact for obesity (where obese and non-obese children corresponded to 0 and 1 , respectively) verses intake ratio of food, exercise, time watching TV and time playing games was assessed using multiple logistic regression where intake ratio of food 1.1 or more and less than 1.1 corresponded to 0 and 1 , exercise yes and no corresponded to 0 and 1 , time watching TV of 2.6 hours or more and less than 2.6 hours corresponded to 0 and 1 , time playing games of 0.3 hours or more and less than 0.3 hours corresponded to 0 and 1 , respectively. Multiple logistic regression of the relation- ship between obesity and exercise was significant (odds ratio $=24.8,95 \%$ confidence interval $=1.2-$ 500.9, $p<0.05$ ) and not significant for other factors.

\section{DISCUSSION}

It was an unexpected finding that the non-obesity group showed a higher intake ratio of food than the obesity group (Table 1). If food-intake is not a primary factor in determining obesity, there may be other factors. We observed that exercise was the key factor to determine obesity. The non-obesity group took less time watching TV and playing games than that of obesity groups. The children with exercise show significantly shorter times of watching TV and playing games. Taking together these results, obesity might not be brought by too much food, but might be brought by non-exercise performance such as watching TV and playing games in children.

In obese children, the intervention of diet control was effective using MNBC but limited as the percentage overweight decreased only from $50 \%$ to $40 \%$ [6]. Since the intervention of obesity was limited by the way of food intake, habitual exercise would determine the obesity. The present non-obese children were volunteer school children and some selections might cause a different result. Surveillance of Japanese school children shows approximately $85 \%$ of primary school children performed exercise and $80 \%$ of junior high school children performed exercise although the percentage of exercise decreased to $60 \%$ in high school students [8]. Of the present non-obese children, $80 \%$ performed exercise

Table 1. Intake-ratio of food in obesity and non-obesity groups.

\begin{tabular}{ccccccccccccc}
\hline & Meat & Fish & Egg & Milk & Beans & $\begin{array}{c}\text { Green and yellow } \\
\text { vegetables }\end{array}$ & Light-colored vegetables & Fruit & Grain & Oil & Sugar & Mean \pm SD \\
\hline Obesity group & 1.0 & 0.6 & 1.4 & 0.5 & 0.8 & 0.3 & 0.5 & 0.9 & 1.5 & 1.4 & 1.4 & $0.9 \pm 0.4$ \\
Non-obesity group & 1.3 & 1.1 & 1.1 & 1.5 & 1.1 & 0.5 & 0.7 & 1.1 & 1.5 & 1.8 & 2.5 & $1.3 \pm 0.4$ \\
& NS & $*$ & NS & $* * *$ & NS & $*$ & $*$ & NS & NS & $* *$ & $* *$ & $* *$ \\
\hline
\end{tabular}

$*(p<0.05), * *(p<0.01)$ and $* * *(p<0.001)$ shows dignificances between the obesity group and non obesity group using the two-sided Mann-Whitney's U test. NS shows no significance. Values are mean \pm SD. 
which seems to be an average percentage for exercise. The present study suggests that in school children, if they exercised, obesity would not be a problem irrespective of the amount of food they took. The present MNBC was proved to be useful for self-care dependent older patients with diabetes mellitus [9]. The subjects were responsible for reporting their own food intake by category but not by the amount of food, which precludes any quantitative estimate of their actual food intake [6]. In adults and older patients with diabetes mellitus, since exercise performance is limited due to social and physical reasons, diet control might be more important in reducing obesity as well as for controlling diabetes mellitus.

\section{REFERENCES}

[1] The Ministry of Education, Culture, Sport, Science and Technology (2009) The nationwide statistics of physical development for Japanese children (in Japanese). Printing Department of the Ministry of Finance, Tokyo.

[2] Flodmark, C.E., Marcus, C. and Britton, M. (2006) Interventions to prevent obesity in children and adolescents, a systematic literature review. International Journal of Obesity, 30, 579-589. doi:10.1038/sj.ijo.0803290

[3] Metcalf, B.S., Voss, L.D., Hosking, J., Jeffery, A.,N. and
Wilkin, T.J. (2008) Physical activity at the government-recommended level and obesity-related health outcomes: A longitudinal study (early bird 37). Archives of Disease in Childhood, 93, 772-777. doi:10.1136/adc.2007.135012

[4] Guillaume, M., Laoidus, L. and Lambert, A. (1998) Obesity and nutrition in children. The belgian luxembourg child study IV. The European Journal Clinical Nutrition, 52, 323-328. doi:10.1038/sj.ejcn.1600532

[5] Wardle, J., Guthrie, C., Sanderson, S., Birch, L. and Plomin, R. (2001) Food and activity preferences in children of lean and obese parents. International Journal of Obesity, 25, 971-977. doi:10.1038/sj.ijo.0801661

[6] Satoh, A., Menzawa, K., Lee, S., Hatakeyama, A. and Sasaki, H. (2007) Dietary guidance for obese children and their families using a model nutritional balance chart. Japan Journal of Nursing Science, 4, 95-102.

[7] Japan Obesity Society (2001) Obesity manual for guidance of obese patients (in Japanese). Ishiyaku Publishers, Tokyo.

[8] Japan School Health Association (2009) Surveillance of health status of Japanese school children (in Japanese). Japan School Health Associationfoundation, Tokyo.

[9] Satoh, A., Sakurada, T., Hatakeyama, A., Fukuoka, Y., Hatakeyama, R. and Sasaki, H. (2008) Dietary guidance for older patients with diabetes mellitus and primary caregivers using a model nutritional balance chart. Japan Journal of Nursing Science, 5, 83-89. doi:10.1111/j.1742-7924.2008.00105.X 